East Asian Mathematical Journal

Vol. 30 (2014), No. 1, pp. 023-029

http://dx.doi.org/10.7858/eamj.2014.003

\title{
PERFORMANCE OF MYOPIC POLICY FOR MULTI-CHANNEL DYNAMIC SPECTRUM ACCESS NETWORKS
}

\author{
YutAE LEE
}

\begin{abstract}
To solve inefficient spectrum usage problem under current static spectrum management policy, various kinds of dynamic spectrum access strategies have appeared. Myopic policy, which maximizes immediate throughput, is a simple and robust strategy with reduced complexity. In this paper, we present a simple mathematical model to evaluate the saturation throughput and medium access delay of a myopic policy in the presence of multiple channels.
\end{abstract}

\section{Introduction}

The exponential growth in wireless services to share the wireless spectrum has recently led to an increasing demand for more bandwidth resources and hence a shortage of them. On the other hand, most spectrum bands suitable for wireless communication have already been allocated to existing licensees $[4,9]$. This expected shortage problem in spectrum supply, which is a major issue for service providers interested in integrating new wireless services into existing communication infrastructure, is reported to be due not to spectrum scarcity, but to current inefficient and static spectrum management policy [11]. Recent extensive measurement-based studies of spectrum efficiency $[2,10]$ suggest that a large portion of the licensed spectrum of deployed wireless communication network lies unused in space and time [12]. As a solution for the inefficient spectrum usage, Federal Communications Commission promotes the so-called dynamic spectrum access, which improves spectrum efficiency by allowing secondary users not having a license for spectrum usage to occupy a spectrum owned by licensee named primary user in a manner that limits interference to primary users $[5,8]$.

Received April 8, 2013; Accepted January 14, 2014.

2000 Mathematics Subject Classification. 60K25, 68M20.

Key words and phrases. queueing theory; multi-channel; myopic policy; dynamic spectrum access.

This research was supported by Basic Science Research Program through the National Research Foundation of Korea(NRF) funded by the Ministry of Education(NRF2013R1A1A4A01013094). 


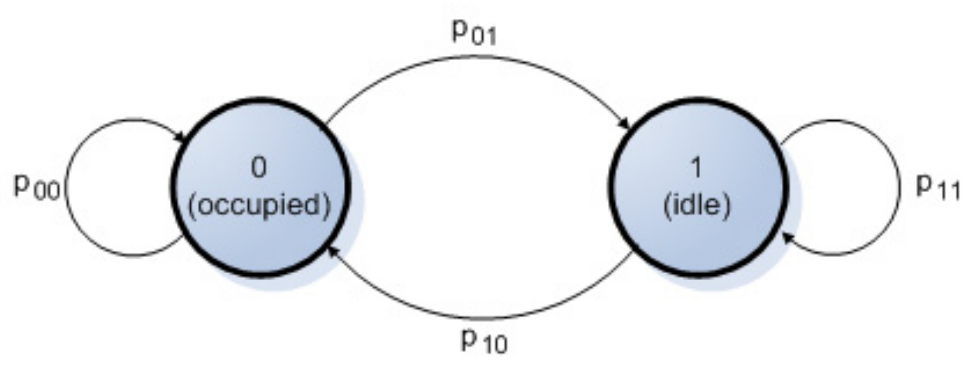

Figure 1. Gilbert-Elliot channel model

A tractable approach for the design of efficient dynamic spectrum access is to consider myopic policies $[1,7,12]$, which aim at maximizing only immediate reward. In $[1,12]$ the throughput of a myopic policy for two channels has been derived in closed-form. The authors also discussed the lower and upper bounds on the throughput of the myopic policy for the case of multiple channels. They show that the myopic policy is a simple and robust strategy that maximizes immediate throughput and achieves optimality under certain conditions [1, 12]. Lee [7] presented a mathematical model for the myopic policy in the presence of only two channels to evaluate the Medium Access Control (MAC) delay as well as the throughput. The MAC delay is an important performance measure when the quality of service of secondary users is considered. To reduce the collision among secondary users, Lee [6] proposed a modified myopic policy with collision avoidance.

In this paper, to compute the throughput and the MAC delay of secondary users for the case of multiple positively correlated channels, we provide a simple and efficient mathematical model for the myopic policy. Based on our model, we derive the throughput performance and the MAC delay distribution of secondary users under saturation traffic conditions.

The rest of the paper is organized as follows. We first study the structure of the myopic policy for the case of multiple positively correlated channels in Section 2 before presenting our mathematical model in Section 3. We also evaluate the performance in Section 3.

\section{Myopic policy}

The myopic policy is shortly summarized in this section. We consider a slotted primary network consisting of $N$ independent and stochastically identical Gilbert-Elliot channels $[3,13]$. As illustrated in Figure 1, the occupancy $S_{i}(k)$, $k \geq 1$ of channel $i, i=1,2, \ldots, N$, at slot $k$ follows a discrete-time two-state 
Markov chain on state space $\{0,1\}$ with one-step transition probability matrix

$$
\mathbf{P}=\left(\begin{array}{ll}
p_{00} & p_{01} \\
p_{10} & p_{11}
\end{array}\right)
$$

State 0 represents that the channel is occupied by primary users while state 1 represents that the channel is idle.

We consider a secondary user seeking spectrum opportunities. Limited by its hardware constraints and energy supply, the secondary user can not observe the full spectrum. However, it can infer the state from its decision and observation history. Immediately before the beginning of slot $k, k \geq 1$, its knowledge of the channels' state is given by its belief vector $\left(\lambda_{1}(k), \lambda_{2}(k), \ldots, \lambda_{N}(k)\right)$, where $\lambda_{i}(k), i=1,2, \ldots, N$, is the conditional probability that channel $i$ is in state 1 immediately before slot $k$, given all past decision and observations. In each slot, if the secondary user has pending packets for transmission, it chooses a channel to sense. For myopic policy, the user chooses an action to maximize expected immediate throughput $[1,7,12]$, i.e., the index $a(k)$ of the channel the user selects at slot $k$ is simply given by

$$
a(k)=\arg \max _{1 \leq i \leq N} \lambda_{i}(k)
$$

for $k \geq 1$. If the chosen channel is sensed to be idle, the secondary user transmits its packet. Otherwise, the secondary user does not transmit the packet. Immediately before the beginning of slot $k+1, k \geq 1$, the belief vector is updated based on the action $a(k)$ with observation outcome $\Theta_{a}(k)$ (indicating the availability of channel $a(k))$ as follows $[1,7,12]$ : for $i=1,2, \ldots, N$

$$
\lambda_{i}(k+1)= \begin{cases}p_{01}, & \text { if } a(k)=i \text { and } \Theta_{a}(k)=0, \\ p_{11}, & \text { if } a(k)=i \text { and } \Theta_{a}(k)=1, \\ \lambda_{i}(k) p_{11}+\left(1-\lambda_{i}(k)\right) p_{01}, & \text { if } a(k) \neq i\end{cases}
$$

We set $\lambda_{i}(1)$ as the stationary probability of the channel being idle, that is,

$$
\lambda_{i}(1)=\frac{p_{01}}{p_{01}+p_{10}},
$$

and choose the initial action randomly.

In this paper, we consider the case of positively correlated channels, that is, $p_{01}<p_{11}$. If channel $i$ is observed to be idle in slot $k$, the belief probability $\lambda_{i}(k+1)$ becomes $p_{11}$. Since $\lambda_{j}(k+1)<p_{11}$ for $j \neq i$, channel $i$ is sensed again in slot $k+1$ (see Figure 2 in [7]). On the other hand, if channel $i$ is observed to be busy in slot $k$, the probability $\lambda_{i}(k+1)$ becomes $p_{01}$. Since $\lambda_{j}(k+1)>p_{01}$ for $j \neq i$, the channel visited the longest time ago is sensed in slot $k+1$ (see Figure 2 in [7]). Thus, in the case of positively correlated channels, the myopic action is to stay in the same channel in the next slot if the channel in the current slot is sensed to be idle. Otherwise, the user switches to the channel visited the longest time ago $[7,12]$. 


\section{Mathematical analysis}

\subsection{Saturation throughput and MAC delay}

We consider a fixed number $N$ of independent and stochastically identical Gilbert-Elliot channels. In saturation traffic conditions, the secondary user always has another packet immediately after the successful completion of a packet transmission. Let $a(k)$ be the stochastic process representing the index of the channel the user selects at slot $k$. Let $s(k)$ be the stochastic process representing the state of the channel $a(k)$ at slot $k$. Clearly, the bi-dimensional process $\{(a(k), s(k)), k \geq 1\}$ is non-Markovian.

We consider the case of positively correlated channels, that is, $p_{01}<p_{11}$. In this case, the key approximation in our model is that the probability $p$ that the switched channel is occupied by primary users is independent on the revisit time of the channel (this is more accurate as $N$ is larger). In this condition, the bi-dimensional process $\{(a(k), s(k)), k \geq 1\}$ is a discrete-time Markov chain with the only non-null one-step transition probabilities being

$$
\begin{aligned}
& P\{a(k+1)=i, s(k+1)=0 \mid a(k)=i, s(k)=1\}=p_{10}, \quad i=1, \cdots, N, \\
& P\{a(k+1)=i, s(k+1)=1 \mid a(k)=i, s(k)=1\}=p_{11}, \quad i=1, \cdots, N, \\
& P\{a(k+1)=i+1, s(k+1)=0 \mid a(k)=i, s(k)=0\}=p, \quad i=1, \cdots, N-1, \\
& P\{a(k+1)=i+1, s(k+1)=1 \mid a(k)=i, s(k)=0\}=1-p, i=1, \cdots, N-1, \\
& P\{a(k+1)=1, s(k+1)=0 \mid a(k)=N, s(k)=0\}=p, \\
& P\{a(k+1)=1, s(k+1)=1 \mid a(k)=N, s(k)=0\}=1-p .
\end{aligned}
$$

Let

$$
\pi_{i, j}=\lim _{k \rightarrow \infty} P\{a(k)=i, s(k)=j\}, \quad i=1, \cdots, N, \quad j=0,1,
$$

be the stationary distribution of the chain. Owing to the chain regularities, the following relations hold:

$$
\begin{aligned}
\pi_{i, 0} & =\pi_{1,0} \\
\pi_{i, 1} & =\frac{1-p}{p_{10}} \pi_{1,0}
\end{aligned}
$$

for $i=1,2, \ldots, N$. The value $\pi_{1,0}$ is determined by imposing the normalization condition

$$
1=\sum_{i=1}^{N}\left(\pi_{i, 0}+\pi_{i, 1}\right)=\pi_{1,0} N\left(1+\frac{1-p}{p_{10}}\right)
$$

from which

$$
\pi_{1,0}=\frac{p_{10}}{N\left(1-p+p_{10}\right)}
$$


The stationary probabilities $\pi_{i, j}$ of $\{(a(k), s(k)), k \geq 1\}$ are given by

$$
\begin{aligned}
\pi_{i, 0} & =\frac{p_{10}}{N\left(1-p+p_{10}\right)}, \\
\pi_{i, 1} & =\frac{1-p}{N\left(1-p+p_{10}\right)}
\end{aligned}
$$

for $i=1,2, \ldots, N$.

The saturation throughput $S_{\text {sat }}$ is given by

$$
S_{\text {sat }}=\sum_{i=1}^{N} \pi_{i, 1}=\frac{1-p}{1-p+p_{10}} .
$$

Now we compute the distribution of the MAC delay of a secondary user under saturation traffic conditions. The MAC delay is defined as the time needed for a packet to be successfully transmitted, given that the packet is at the head-of-line position in the buffer. Let $d_{n}, n=1,2, \ldots$, be the probability that the MAC delay under saturation traffic condition is $n$ slots. Then

$$
\begin{aligned}
& d_{1}=p_{11} \\
& d_{n}=p_{10} p^{n-2}(1-p), \quad n=2,3, \ldots
\end{aligned}
$$

\subsection{Determination of unknown probability $p$}

To finally compute the probability $p$, we define $T_{i}$ as the random variable representing the number of time slots that the secondary user stays continuously in channel $i$. Assuming that the probability $p$ is independent on the recurrent time of a channel, we obtain

$$
P\left\{T_{i}=n\right\}= \begin{cases}p, & n=1 \\ (1-p)\left(1-p_{10}\right)^{n-2} p_{10}, & n=2,3, \cdots,\end{cases}
$$

and its $z$-transform

$$
T_{i}(z)=E\left[z^{T_{i}}\right]=\sum_{n=1}^{\infty} P\left\{T_{i}=n\right\} z^{n}=\frac{p z(1-z)+p_{10} z^{2}}{1-\left(1-p_{10}\right) z} .
$$

Let $T$ be the number of time slots that the secondary user stays continuously in $N-1$ different channels, without loss of generality

$$
T=T_{1}+\cdots+T_{N-1} .
$$

Since $T_{i}$ 's are independent and identically distributed, the $z$-transform $T(z)$ of $T$ can be obtained as

$$
T(z)=E\left[z^{T}\right]=\left[T_{1}(z)\right]^{N-1}=\left[\frac{p z(1-z)+p_{10} z^{2}}{1-\left(1-p_{10}\right) z}\right]^{N-1} .
$$


Note that $p$ is the probability that the switched channel is occupied by primary users. Thus

$$
p=\sum_{n=N-1}^{\infty} P\{T=n\} p_{00}^{(n+1)}
$$

where $p_{00}^{(n)}$ is the $n$-step transition probability from state 0 to state 0 , i.e., $p_{00}^{(n)}=P\left\{S_{i}(n)=0 \mid S_{i}(0)=0\right\}$. The probability $p_{00}^{(n)}$ is obtained as follows. Let

$$
\mathbf{P}^{(n)}=\left(p_{i j}^{(n)}\right)
$$

be the $n$-step transitional probability matrix, which satisfies

$$
\mathbf{P}^{(n+1)}=\mathbf{P}^{(n)} \cdot \mathbf{P}
$$

Defining the matrix generating function of $\mathbf{P}^{(n)}$ 's as

$$
\mathbf{P}(z)=\sum_{n=0}^{\infty} \mathbf{P}^{(n)} z^{n}
$$

we obtain

$$
\mathbf{P}(z)=(\mathbf{I}-z \mathbf{P})^{-1} .
$$

From the inverse-transform of $\mathbf{P}(z)$, we obtain $\mathbf{P}^{(n)}$ as follows:

$\mathbf{P}^{(n)}=\left(\begin{array}{cc}\frac{p_{10}}{p_{01}+p_{10}} & \frac{p_{01}}{p_{01}+p_{10}} \\ \frac{p_{10}}{p_{01}+p_{10}} & \frac{p_{01}}{p_{01}+p_{10}}\end{array}\right)+\left(1-p_{10}-p_{01}\right)^{n}\left(\begin{array}{cc}\frac{p_{01}}{p_{01}+p_{10}} & -\frac{p_{01}}{p_{01}+p_{10}} \\ -\frac{p_{10}}{p_{01}+p_{10}} & \frac{p_{10}}{p_{01}+p_{10}}\end{array}\right)$

Hence, we obtain

$$
p_{00}^{(n)}=\frac{p_{10}}{p_{01}+p_{10}}+\left(1-p_{10}-p_{01}\right)^{n} \frac{p_{01}}{p_{01}+p_{10}} .
$$

Using (13) and (19), (14) becomes

$$
\begin{aligned}
p= & \sum_{n=N-1}^{\infty} P\{T=n\}\left[\frac{p_{10}}{p_{01}+p_{10}}+\left(p_{11}-p_{10}\right)^{n+1} \frac{p_{01}}{p_{01}+p_{10}}\right] \\
= & \frac{p_{10}}{p_{01}+p_{10}}+\frac{p_{01}}{p_{01}+p_{10}}\left(p_{11}-p_{10}\right) T\left(p_{11}-p_{10}\right) \\
= & \frac{p_{10}}{p_{01}+p_{10}}+\frac{p_{01}}{p_{01}+p_{10}}\left(p_{11}-p_{10}\right) \\
& \times\left[\frac{p\left(p_{11}-p_{10}\right)\left(p_{10}+p_{01}\right)+p_{10}\left(p_{11}-p_{10}\right)^{2}}{1-\left(1-p_{10}\right)\left(p_{11}-p_{10}\right)^{2}}\right]^{N-1} .
\end{aligned}
$$

Now, we show that (20) has unique solution in $0 \leq p \leq 1$. Defining

$$
\begin{aligned}
f(p) & =\frac{p_{01}+p_{10}}{p_{01}\left(p_{11}-p_{10}\right)}\left[p-\frac{p_{10}}{p_{01}+p_{10}}\right] \\
g(p) & =\left[\frac{\left(p_{11}-p_{10}\right)\left(p_{10}+p_{01}\right)}{1-\left(1-p_{10}\right)\left(p_{11}-p_{10}\right)} p+\frac{p_{10}\left(p_{11}-p_{10}\right)^{2}}{1-\left(1-p_{10}\right)\left(p_{11}-p_{10}\right)}\right]^{N-1}
\end{aligned}
$$


Eq. (20) becomes $f(p)=g(p)$. Note that $f(0)<0, f(1)>1$, the coefficient of $p^{N-1}$ in $g(p)$ is positive, and $g(p *)=0$ for $p *=-p_{10}\left(1-p_{01}-p_{10}\right) /\left(p_{01}+p_{10}\right)<$

0 . Furthermore, since

$$
g(1)=\left[\frac{\left(1-p_{10}-p_{01}\right)\left(p_{10}+p_{01}\right)+p_{10}\left(1-p_{10}-p_{01}\right)^{2}}{1-\left(1-p_{10}\right)\left(1-p_{10}-p_{01}\right)^{2}}\right]^{N-1}<1,
$$

Eq. (20) has unique solution in $0 \leq p \leq 1$.

\section{References}

[1] S. H. Ahmad, M. Liu, T. Javadi, Q. Zhao, and B. Krishnamachari, Optimality of myopic sensing in multi-channel opportunistic access, IEEE Trans. Information Theory $\mathbf{5 5}$ (2009), no. 9, 4040-4050.

[2] S. Geirhofer, L. Tong, and B. M. Sadler, Dynamic spectrum access in the time domain: modeling and exploiting white space, IEEE Commun. Mag. 45 (2007), no. 5, 66-72.

[3] E. N. Gilbert, Capacity of burst-noise channels, Bell Syst. Tech. J. 39 (1960), 1253-1265.

[4] S. Huang, X. Liu, and Z. Ding, Opportunistic spectrum access in cognitive radio networks, Proc. IEEE INFOCOM 2008, 2008

[5] Y. Lee, Opportunistic spectrum access in cognitive networks, Electronics Letters 44 (2008), no. 17, 1022-1024.

[6] Modified myopic policy with collision avoidance for opportunistic spectrum access, Electronics Letters 46 (2010), no. 12, 871-872.

[7] _ Performance of myopic policy for opportunistic spectrum sharing, East Asian Mathematical Journal 27 (2011), no. 1, 23-33.

[8] M. M. Rashid, Md. J. Hossain, E. Hossain, and V. K. Bhargava, Opportunistic spectrum access in cognitive radio networks: a queueing analytic model and admission controller design, Proc. IEEE GLOBECOM 2007 (2007), 4647-4652.

[9] A. Sabharwal, A. Khoshnevis, and E. Knightly, Opportunistic spectral usage: bounds and a multi-band CSMA/CA protocol, IEEE/ACM Transactions on Networking (2007), 533-545.

[10] Shared Spectrum Company, Reports on spectrum occupancy measurements, http://www.sharedspectrum.com/measurements/recent.html.

[11] M. Vilimpoc and M. McHenry, Dupont circle spectrum utilization during peak hours, http://www.newamerica.net/files/archive/Doc_File_183_1.pdf, 2006.

[12] Q. Zhao, B. Krishnamachari, and K. Liu, On myopic sensing for multi-channel opportunistic access: structure, optimality, and performance, IEEE Trans. Wireless Communications 7 (2008), no. 12.

[13] M. Zorzi, R. Rao, and L. Milstein, Error statistics in data transmission over fading channels, IEEE Trans. Commun. 46 (1998), 1468-1477.

Yutae LeE

Department of Information and Communication EngineERing

Dongeui University

BUSAN 614-714, Korea

E-mail address: ylee@deu.ac.kr 